\title{
Multipoint Fluorescence Quenching-Time Statistics for Single Molecules with Anomalous Diffusion
}

\author{
Valeri Barsegov ${ }^{\dagger}$ and Shaul Mukamel $*,+,,, \S$ \\ Departments of Chemistry and Physics and Astronomy, University of Rochester, \\ Rochester, New York 14627-0216
}

Received: May 30, 2003; In Final Form: August 25, 2003

\begin{abstract}
Three-point fluorescence lifetime correlation functions are computed for the fluorescence resonance energy transfer (FRET) and electron transfer (ET) quenching mechanisms in a single donor-acceptor system of which the distance $X$ undergoes anomalous diffusion in a harmonic potential with short-time variance scaling as $\sim t^{\alpha}$. The three-point joint probability distribution of $X$ and its moments are calculated by solving the fractional Fokker-Planck equation (FFPE). For $\alpha=1$, the process is stationary and the two- and three-point joint probability distribution is centered around the time-dependent average donor-acceptor separation, $\langle X(t)\rangle$. For $\alpha<1$, the distribution slows down, becomes nonstationary, and remains centered at initial separation $X(0)$ even for times exceeding the bath correlation time scale.
\end{abstract}

\section{Introduction}

Single-molecule spectroscopy (SMS) has the capacity to monitor the entire distributions of various molecular properties, extracting dynamical information not accessible from bulk measurements. ${ }^{1-4}$ The dynamics of, for example, conformational motions of proteins spans many decades of time scales ranging from femtoseconds to seconds. While ensemble-averaged experiments have been used to probe fast conformational fluctuations compared to some internal clock, for example, rotational diffusion in fluorescence depolarization or fluorescence lifetime in Stokes shift measuremnents, ${ }^{5}$ it is hard to find suitable clocks for slow molecular motions (e.g., conformational fluctuations of large subdomains). Furthermore, bulk measurements cannot tell whether all molecules have the same distribution or each molecule makes a distinct contribution to that distribution. SMS allows one to probe slow motions by studying one molecule at a time.

Much theoretical work has been devoted recently to study of dynamical disorder on a broad range of time scales at the singlemolecule level. ${ }^{6-12}$ In probing dynamic disorder using optical techniques, ${ }^{13}$ most common observables are autocorrelation functions of the chromophore absorbing frequency, ${ }^{14}$ fluorescence intensity, ${ }^{13}$ fluorescence lifetime fluctuations, ${ }^{15}$ and duration of on-time events (i.e., time during which a single molecule is in a fluorescence active state). ${ }^{16,17}$ The dynamics of correlations of bath variables can also be probed by photon statistics by examining, for example, the Mandel parameter, which describes deviations of the distribution of number of emitted photons from Poissonian. ${ }^{15,18-20}$

Fluorescence resonance energy transfer (FRET) measurements between single pairs of donor and acceptor fluorophores provide information about structure and distance fluctuations of a single biomolecule (RNA, DNA, enzymes) or between components

\footnotetext{
Department of Chemistry.

$\doteqdot$ Department of Physics and Astronomy.

$\S$ Present Address: Department of Chemistry, University of California, Irvine, Irvine, CA 92697-2025. E-mail: smukamel@uci.edu.
}

of interacting biomolecules (e.g., lifetime of an enzymesubstrate contact, opening and closing of ion channels in a membrane). ${ }^{21-24}$ FRET data reflect conformational states in the molecular center-of-mass frame and are not complicated by overall translocations or rotations. FRET measurements on a single molecule can access conformational subpopulations and dynamics, ligand binding, kinetics of folding/unfolding and protein aggregation, and enzyme catalysis. FRET has recently been used to probe conformational dynamics of staphylococcal nuclease (SNase) and catalytic turnovers of DNA and RNA hydrolysis into mono- and dinucleotides. ${ }^{16,21,22,25}$

Both discrete-jump and continuous models have been used in recent studies of dynamically fluctuating environments of a single molecule. Xie and co-workers have studied the distribution of jump rates by following the closing and opening of single-stranded DNA hairpins and observed multiexponential decay of the two-time correlation functions of fluorescence lifetime fluctuation. ${ }^{26}$ The overdamped Brownian oscillator is a widely used model suitable for the interpretation of spectroscopic measurements, in which the stochastic bath evolution is described by a continuous variable. ${ }^{27}$

For ordinary diffusion with no external potential, the meansquare fluorophore-quencher distance exhibits linear scaling in time, $\left\langle X^{2}\right\rangle \approx D t$, where $D$ is diffusion coefficient. However, systems with complex potential energy landscapes, for example, glasses, supercooled liquids, and biomolecules, have more elaborate kinetics. ${ }^{17,28,29}$ In these systems, conformational relaxation follows anomalous subdiffusive dynamics in which $\left\langle X^{2}\right\rangle \approx t^{\alpha}$ with $0<\alpha<1$ at short times.

Recently, Metzler, Barkai, and Klafter ${ }^{30,31}$ have studied the subdiffusive dynamics of a harmonically bound particle using a fractional Fokker-Planck equation (FFPE) approach, which uses fractional derivatives. ${ }^{32}$ They have computed the Green function, $W\left(X_{1}, t_{1} ; X_{0}, t_{0}\right)$, of a subdiffusive particle evolving on the harmonic potential. ${ }^{30,31}$ Yang and Xie used this Green function to compute the two-time correlation functions of 
fluorescence lifetime fluctuations $\langle\delta \tau(t) \delta \tau(0)\rangle$ for FRET and ET quenching mechanisms for a broad range of subdiffusive model parameters and compared it with correlators of lifetime fluctuations due to Brownian motion. ${ }^{17}$ By fitting the short time limit of Mittag-Leffler-type subdiffusive relaxation of $\langle\delta \tau(t) \delta \tau(0)\rangle$ given by a stretched exponential, that is, $\langle\delta \tau(t) \delta \tau(0)\rangle \approx \exp \left[-k t^{\alpha}\right]$, into correlators obtained from computer simulation of ET quenching, they obtained the stretching parameter $\alpha=0.2$.

In this paper, we consider both FRET quenching mechanism in which the rate varies as $\sim X^{-6}$ and ET quenching with the rate varying as $\exp (-X)$. We study the multitime correlations of the fluorescence lifetime by computing the three-point joint distribution of the donor-acceptor distance and the three-time correlation function $\left\langle\tau\left(t_{2}\right) \tau\left(t_{1}\right) \tau\left(t_{0}\right)\right\rangle$ of the fluorescence lifetime $\tau$ using the overdamped Brownian-oscillator model for the donor-acceptor distance $X$. This readily available experimental observable reflects fluctuations of $X$ that determine the fluorescence quenching rate. Higher-order correlations of dynamical variables contain increasingly more detailed information about the dynamics of the environment variables and thus provide critical tests for theoretical models. ${ }^{27,33}$

The model is presented in section II. In section III, we derive expressions for the forward and backward propagators for the fractional Fokker-Planck equation and compute the two-point conditional probability and the three-point joint probability of the separation coordinate $X$ for $\alpha=0.5$ and $\alpha=1$. The threepoint joint distributions are computed in section IV, and the three-time correlation function of the fluorescence lifetime for FRET and ET quenching mechanisms is computed in section $\mathrm{V}$. Technical details are given in the appendices.

\section{The Model}

We consider a fluorophore (donor chromophore) and fluorescence quencher (acceptor) attached to a polymer. The fluorescence lifetime is $\tau=\left(\gamma_{0}+\gamma_{\mathrm{q}}\right)^{-1}$, where $\gamma_{0}$ is the intrinsic (radiative and nonradiative) fluorescence decay rate of the donor and $\gamma_{\mathrm{q}}$ is the quenching rate. Conformational fluctuations alter $\gamma_{\mathrm{q}}$ by varying the donor-acceptor distance $X(t)$, and the fluorescence lifetime $\tau(t)=\left[\gamma_{0}+\gamma_{\mathrm{q}}^{j}(X(t))\right]^{-1}(j=$ FRET or ET) becomes a stochastic quantity. We assume that the conformational fluctuations are slower than the average fluorescence lifetime.

In FRET, the time-dependent quenching rate is ${ }^{34,35}$

$$
\gamma_{\mathrm{q}}^{\mathrm{FRET}}(t) \approx \gamma_{0}\left(\frac{R_{0}}{X(t)}\right)^{6}
$$

where $R_{0}$ is the Foerster radius. Another mechanism of fluorescence quenching is photoinduced ET in which the quenching rate depends exponentially on the donor-acceptor distance, that is,

$$
\gamma_{\mathrm{q}}^{\mathrm{ET}}(t)=k_{0} \mathrm{e}^{-\beta X(t)}
$$

The two-time correlation function of fluorescence lifetime,

$$
C\left(t_{1}, t_{0}\right) \equiv\left\langle\tau\left(t_{1}\right) \tau\left(t_{0}\right)\right\rangle
$$

where the angular bracket, $\langle\ldots\rangle$, denotes ensemble average, may be computed as

$$
\begin{aligned}
& \left\langle\tau\left(t_{1}\right) \tau\left(t_{0}\right)\right\rangle= \\
& \quad \int_{-\infty}^{\infty} \mathrm{d} X_{1} \int_{-\infty}^{\infty} \mathrm{d} X_{0} \tau\left(X_{1}\right) W\left(X_{1}, t_{1} ; X_{0}, t_{0}\right) \tau\left(X_{0}\right) \rho_{\mathrm{eq}}\left(X_{0}\right)
\end{aligned}
$$

where $\rho_{\mathrm{eq}}(X)$ is the equilibrium distribution of $X$ defined in eq 21 and $W\left(X_{1}, t_{1} ; X_{0}, t_{0}\right)$ is the conditional probability to find the coordinate in $X_{1}$ at time $t_{1}$ given that it was in $X_{0}$ at an earlier time $t_{0}<t_{1}$. It connects the probability distribution of $X, P(X, t)$, at these two times, that is,

$$
P\left(X_{1}, t_{1}\right)=\int_{-\infty}^{\infty} \mathrm{d} X_{0} W\left(X_{1}, t_{1} ; X_{0}, t_{0}\right) P\left(X_{0}, t_{0}\right)
$$

In many systems with complex free-energy landscapes, the mean-square fluorophore-quencher displacement deviates from linear scaling, and conformational relaxation undergoes anomalous subdiffusion in which $\left\langle\Delta X^{2}\right\rangle \approx t^{\alpha}$, where $0<\alpha<1 .{ }^{17,28,29}$ Subdiffusive dynamics of an overdamped coordinate can be described using a fractional Fokker-Planck equation for Ornstein-Uhlenbeck process, developed by Metzler, Barkai, and Klafter. ${ }^{30,31,32}$ The one-dimensional FFPE for the distribution $W_{\alpha}\left(X_{1}, t_{1} ; X_{0}, 0\right)$ subject to the initial condition $W_{\alpha}\left(X_{1}, 0 ; X_{0}, 0\right)=$ $\delta\left(X_{1}-X_{0}\right)$ is given by

$$
\dot{W}_{\alpha}\left(X_{1}, t_{1} ; X_{0}, 0\right)={ }_{0} D_{t_{1}}^{1-\alpha} L_{\mathrm{FP}}\left(X_{2}\right) W_{\alpha}\left(X_{1}, t_{1} ; X_{0}, 0\right)
$$

where the Fokker-Planck differential operator,

$$
L_{\mathrm{FP}}=\frac{\partial V^{\prime}(X)}{\partial X m \eta_{\alpha}}+K_{\alpha} \frac{\partial^{2}}{\partial X^{2}}
$$

depends on the generalized diffusion $K_{\alpha}=k_{\mathrm{B}} T /\left(m \eta_{\alpha}\right)$ and friction $\eta_{\alpha}$ constants and $V^{\prime}(X)$ is the gradient of the external potential $V(X)=m \omega^{2} X^{2} / 2$.

The Riemann-Liouville fractional integro-differential operator appearing on the right-hand side of eq 6 is ${ }^{32}$

$$
{ }_{0} D_{t_{1}}{ }^{1-\alpha} W_{\alpha}\left(X_{1}, t_{1}\right) \equiv \frac{1}{\Gamma(\alpha)} \frac{\partial}{\partial t_{1}} \int_{t_{0}}^{t_{1}} \mathrm{~d} t^{\prime} \frac{W_{\alpha}\left(X_{1}, t^{\prime}\right)}{\left(t_{1}-t^{\prime}\right)^{1-\alpha}}
$$

where we suppressed the initial state variables $X_{0}$ and $t_{0}$ in $W_{\alpha}$. This operator introduces a convolution integral with a power-law kernel $1 /\left(t-t^{\prime}\right)^{1-\alpha}$ typical for memory effects in condensed phases with complex potentials. When $\alpha=1$, the FFPE reduces to the standard Fokker-Plank equation (FPE) and the evolution of $X$ is described by the Langevin equation, $\dot{X}(t)=-\lambda_{1} X(t)+f(t)$, where $\lambda_{1}=\omega^{2} / \eta_{1}$ is the drift coefficient, the inverse of which determines the time scale of correlation of $X$, and the random force $f(t)$ is assumed to be a Gaussian white noise, $\left\langle f(t) f\left(t^{\prime}\right)\right\rangle=2 \lambda_{1} \theta \delta\left(t-t^{\prime}\right)$, where $\theta \equiv k_{\mathrm{B}} T /\left(m \omega^{2}\right)$ is the magnitude of fluctuations with zero mean. For more insight on the physical origin of the parameters $K_{\alpha}$ and $\eta_{\alpha}$ appearing in eqs 6 and 7, we refer the reader to ref 36 . 

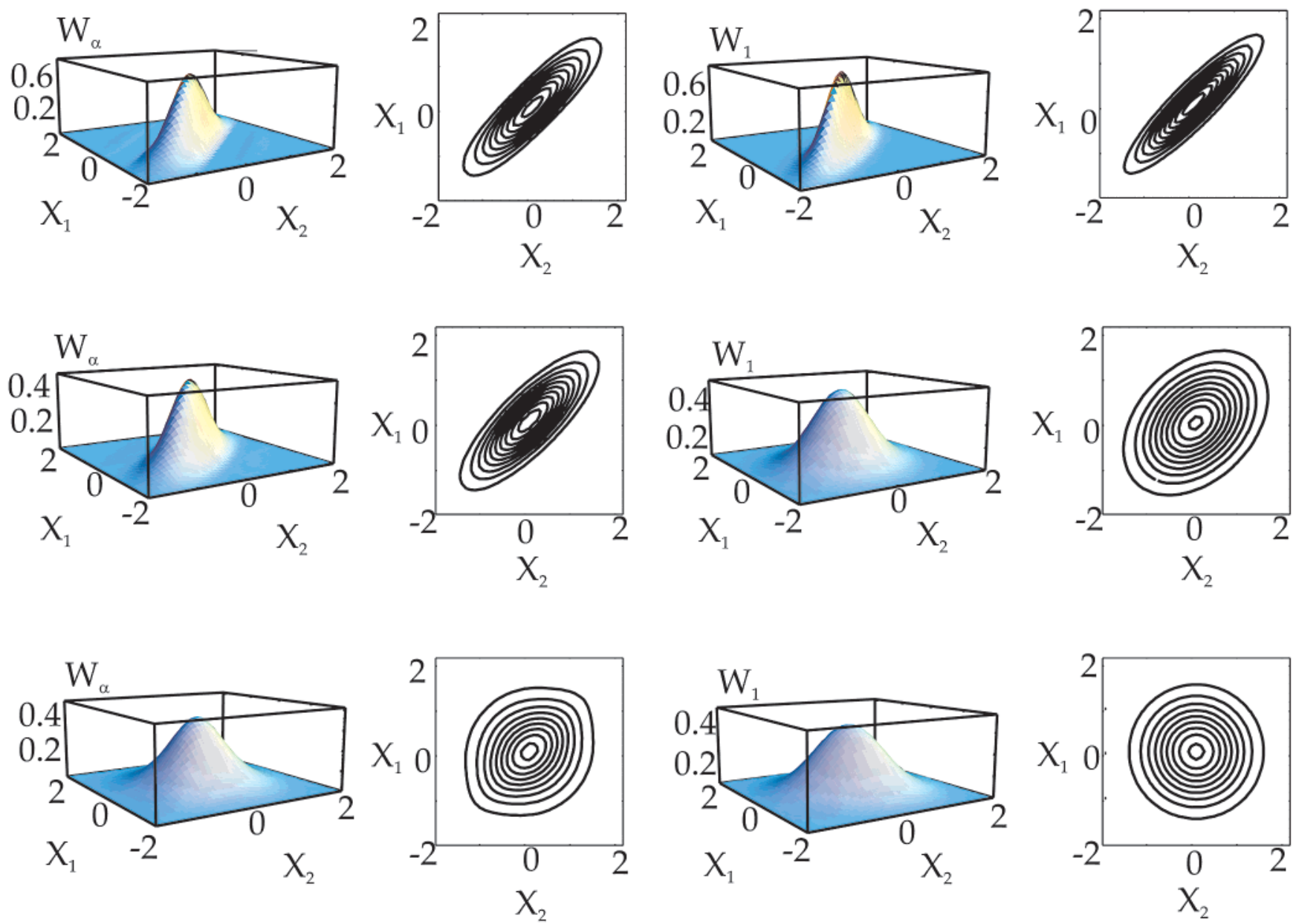

Figure 1. The probability $W_{\alpha}\left(X_{2}, \bar{t}_{2} ; X_{1}, \bar{t}_{1}\right) \rho_{\mathrm{eq}}\left(X_{1}\right)$ vs $X_{1}$ and $X_{2}(\AA)$ for models $\mathrm{M} 1$ (left) and M2 (right) for $\bar{t}_{1}=1.0 \mathrm{ps}$ and $\bar{t}_{2}=1.0 \mathrm{ps}$ (top), $\bar{t}_{1}=$ $1.0 \mathrm{ps}$ and $\bar{t}_{2}=10.0 \mathrm{ps}$ (middle), and $\bar{t}_{1}=1.0 \mathrm{ps}$ and $\bar{t}_{2}=100 \mathrm{ps}$ (bottom). Contour plots are shown to the right of 2D surfaces.

\section{Forward versus Backward Green Functions for FFPE}

The forward propagator $W^{+}$can be expanded in a complete set of eigenstates $\left\{\phi_{n}(X)\right\}$ of the FFPE $(t \geq 0)^{28,29,31}$

$$
\begin{aligned}
W_{\alpha}^{+}( & \left.X_{1}, t_{1} ; X_{0}, t_{0}\right) \\
& =E_{\alpha}\left(L_{\mathrm{FP}}\left(X_{1}\right) t_{10}^{\alpha}\right) \delta\left(X_{1}-X_{0}\right) \\
& =\mathrm{e}^{\tilde{V}\left(X_{0}\right)} \sum_{n=0}^{\infty} E_{\alpha}\left(L_{\mathrm{FP}}\left(X_{1}\right) t^{\alpha}\right) \phi_{n}\left(X_{1}\right) \phi_{n}\left(X_{0}\right) \\
& =\mathrm{e}^{\tilde{V}\left(X_{0}\right)} \sum_{n=0}^{\infty} \phi_{n}\left(X_{1}\right) \phi_{n}\left(X_{0}\right) E_{\alpha}\left(-\lambda_{n, \alpha} t_{10}^{\alpha}\right) \\
& =\mathrm{e}^{\tilde{V}\left(X_{0}\right) / 2-\tilde{V}\left(X_{1}\right) / 2} \sum_{n=0}^{\infty} \psi_{n}\left(X_{1}\right) \psi_{n}\left(X_{0}\right) E_{\alpha}\left(-\lambda_{n, \alpha} t_{10}^{\alpha}\right)
\end{aligned}
$$

where here and hereafter $t_{i j} \equiv t_{i}-t_{j}$, and the functions $\psi_{n}(X)=\phi_{n}(X) \exp [\tilde{V}(X) / 2]$ are related to the eigenfunctions of $L_{\mathrm{FP}}, \phi_{n}(X)$, through the scaled potential $\tilde{V}(X)=V(X) /\left[k_{\mathrm{B}} T\right]$,

$$
\psi_{n}(X)=\left[\frac{1}{2 \pi \theta}\right]^{1 / 4} \frac{1}{\sqrt{2^{n} n !}} H_{n}(\tilde{X} / \sqrt{2}) \mathrm{e}^{-\tilde{X}^{2} / 2}
$$

$\left\{\psi_{n}(X)\right\}$ form an orthonormal basis set, that is,

$$
\int_{-\infty}^{\infty} \mathrm{d} X \psi_{n}(X) \psi_{m}(X)=\int_{-\infty}^{\infty} \mathrm{d} X \mathrm{e}^{\tilde{V}(X)} \phi_{n}(X) \phi_{m}(X)=\delta_{n m}
$$

The eigenvalues $\lambda_{n, \alpha}=n \omega^{2} / \eta_{\alpha}, n=0,1,2$, etc., are related to the eigenvalues $\lambda_{n, 1}$ of the standard Fokker-Planck equation by a dimensionless rescaling factor $\lambda_{n, \alpha}=\left[\eta_{1} / \eta_{\alpha}\right] \lambda_{n, 1}$.

Substituting eq 10 in eq 9 gives $^{30,31}$

$$
\begin{aligned}
& W_{\alpha}^{+}\left(X_{1}, t_{1} ; X_{0}, t_{0}\right)= \\
& \quad \frac{1}{\sqrt{2 \pi} \theta^{n=0} 2^{n} n !} \sum_{\alpha}^{\infty} \frac{1}{}\left(-n \tilde{t}_{10}^{\alpha}\right) H_{n}\left(\tilde{X}_{0} / \sqrt{2}\right) H_{n}\left(\tilde{X}_{1} / \sqrt{2}\right) \mathrm{e}^{-\tilde{X}_{1}^{2} / 2}
\end{aligned}
$$

where $\tilde{X}=X / \sqrt{\theta}, \tilde{t}=t\left(\lambda_{\alpha}\right)^{1 / \alpha}$, and $H_{n}$ are Hermite polynomials with eigenvalues $\lambda_{n, \alpha}=n \lambda_{\alpha} . E_{\alpha}$ is the Mittag-Leffler function

$$
E_{\alpha}\left(-n \tilde{t}^{\alpha}\right)=\sum_{m=0}^{\infty} \frac{\left(-n \tilde{t}^{\alpha}\right)^{m}}{\Gamma(1+m \alpha)}
$$

It crosses over between a stretched exponential at short 

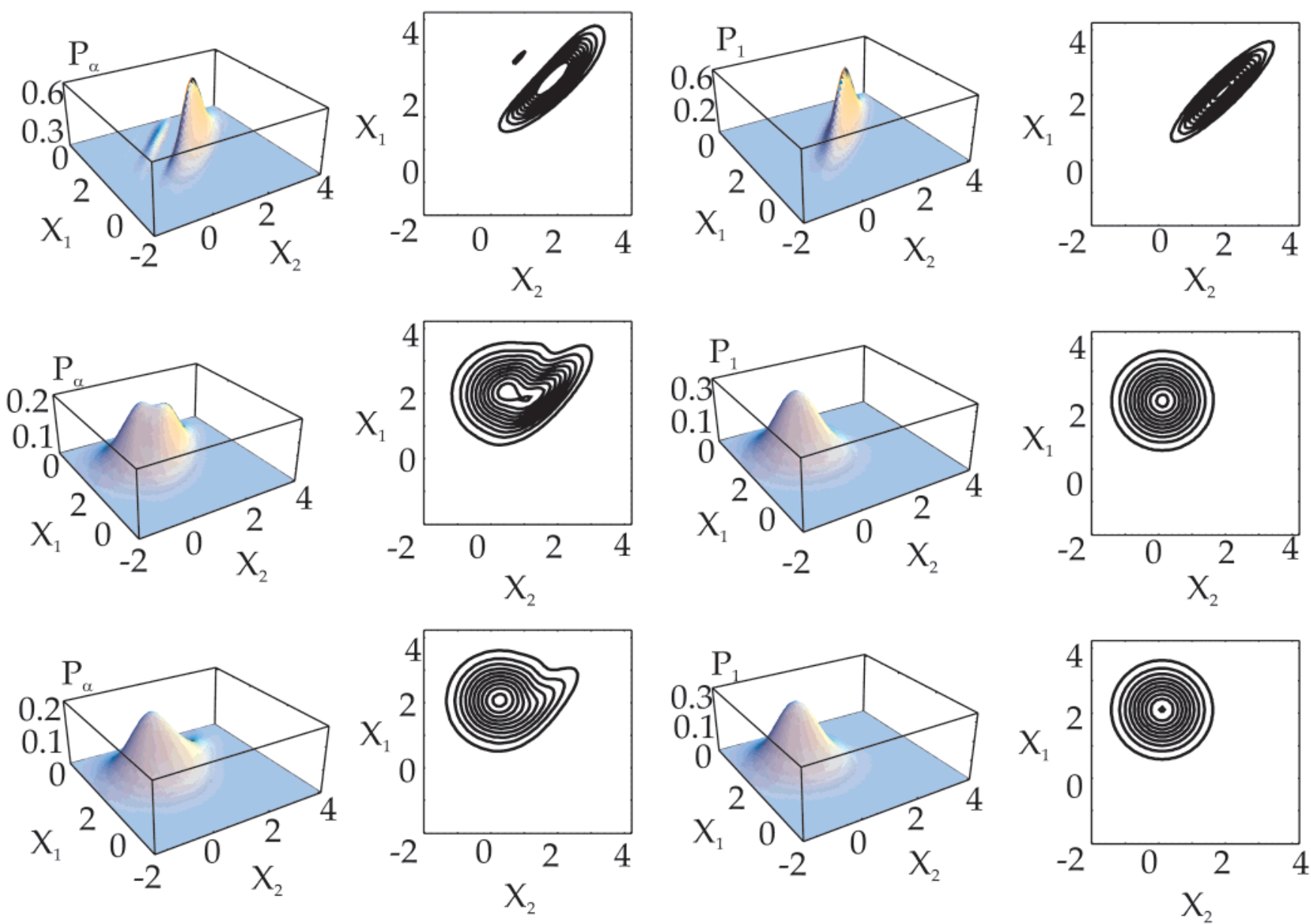

Figure 2. The joint probability $P_{\underline{\alpha}}\left(X_{2}, \bar{t}_{2} ; X_{1}, \bar{t}_{1}\right)$ vs $X_{1}$ and $X_{2}$ ( $\AA$ ) for models M3 (left) and M4 (right) for $\bar{t}_{1}=1.0 \mathrm{ps}$ and $\bar{t}_{2}=1.0 \mathrm{ps}$ (top), $\bar{t}_{1}=1.0$ ps and $\bar{t}_{2}=200 \mathrm{ps}$ (middle), and $\bar{t}_{1}=1.0 \mathrm{ps}$ and $\bar{t}_{2}=1.5 \mathrm{~ns}$ (bottom). Contour plots are shown to the right of 2D surfaces.

times compared to $\left(\lambda_{\alpha}\right)^{-1 / \alpha}$,

$$
E_{\alpha}\left(-n \tilde{t}^{\alpha}\right) \approx \exp \left[-\frac{n \lambda_{\alpha} t^{\alpha}}{\Gamma(1+\alpha)}\right]
$$

and a power law at long times,

$$
E_{\alpha}\left(-n \tilde{t}^{\alpha}\right) \approx\left[n \lambda_{\alpha} \Gamma(1-\alpha)\right]^{-1} t^{-\alpha}
$$

where $\Gamma(z)=\int_{0}^{\infty} \mathrm{d} y y^{z-1} \mathrm{e}^{-y}$ is the gamma function.

For $\alpha=1$, the Mittag-Leffler function becomes a simple exponential, and we recover the solution of the ordinary FPE,

$$
\begin{aligned}
& W_{1}^{+}\left(X_{1}, t_{1} ; X_{0}, t_{0}\right)= \\
& \frac{1}{\sqrt{2 \pi \theta\left(1-\mathrm{e}^{-2 \lambda_{1} t_{10}}\right)}} \exp \left(-\frac{\left(X_{1}-X_{0} \mathrm{e}^{-\lambda_{1} t_{10}}\right)^{2}}{2 \theta\left(1-\mathrm{e}^{-2 \lambda_{1} t_{10}}\right)}\right)
\end{aligned}
$$

where we used the summation formula for the Hermite polynomials. ${ }^{37,38}$

Equation 12 can only be used to compute the two-time correlation functions $\left\langle A(X(t)) A\left(X\left(t_{0}\right)\right)\right\rangle$ of various dynamical quantities when the first time is $t_{0}$. In the continuous time random walk (CTRW) approach to Brownian diffusion, the waiting time distribution function, $w(t)$, for successive jumps is Poissonian, that is, $w(t)=\langle t\rangle^{-1} \exp [-t /\langle t\rangle]$, where $\langle t\rangle$ is the average time between successive jumps. ${ }^{39-43}$ In CTRW, all walkers have arrived at $X_{0}$ exactly at time $t_{0}$. In the fractal time random walk, the system does not equilibrate and retains memory of the initial time, which results in the long-tailed waiting time distribution, $w(t) \approx(\langle t\rangle / t)^{1+\alpha} \cdot{ }^{30,31}$ Because of memory effects (represented by the power-law kernel in the Riemann-Liouville operator (eq 8)), the distribution of waiting times underlying $X$ at time $t_{0}$ differs from the distribution at later times $t>0$. As a result, the process becomes nonMarkovian, that is, the current distribution of $X$ alone is not sufficient to predict the future distribution and more information is needed. However, the process is Markovian but only for a special initial time $t_{0}$, and to compute multitime correlation functions, we need a general Green function $W_{\alpha}\left(X_{2}, t_{2} ; X_{1}, t_{1}\right)$ for arbitrary time $t_{1} \neq t_{0}$. This function can be constructed if we go back and forth to this special time $t_{0}$, that is, by propagating the system from $X_{1}$ at $t_{1}$ back in time to $X_{0}$ at time $t_{0}$, followed by forward propagation to state $X_{2}$ at time $t_{2}$, that is,

$$
W_{\alpha}\left(X_{2}, t_{2} ; X_{1}, t_{1}\right)=\int_{-\infty}^{\infty} \mathrm{d} X_{0} W_{\alpha}^{+}\left(X_{2} t_{2} ; X_{0} t_{0}\right) W_{\alpha}^{-}\left(X_{0} t_{0} ; X_{1} t_{1}\right)
$$

The backward propagator, ${ }_{0} D_{t}{ }^{1-\alpha} L_{\mathrm{FP}}^{\dagger}(X)$, is computed in Appendix A by solving the backward FFPE. Substituting the forward $W_{\alpha}^{+}$and backward $W_{\alpha}^{-}$propagators given by eqs 9 and 29 in eq 17 , we obtain $\left(t_{2} \geq t_{1}\right)$

$$
\begin{aligned}
& W_{\alpha}\left(X_{2}, t_{2} ; X_{1}, t_{1}\right)= \\
& \mathrm{e}^{\tilde{V}\left(X_{1}\right) / 2-\tilde{V}\left(X_{2}\right) / 2} \sum_{n=0}^{\infty} \psi_{n}\left(X_{2}\right) \psi_{n}\left(X_{1}\right) E_{\alpha}\left(-\lambda_{n, \alpha} t_{20}^{\alpha}\right) E_{\alpha}\left(\lambda_{n, \alpha} t_{10}^{\alpha}\right)
\end{aligned}
$$



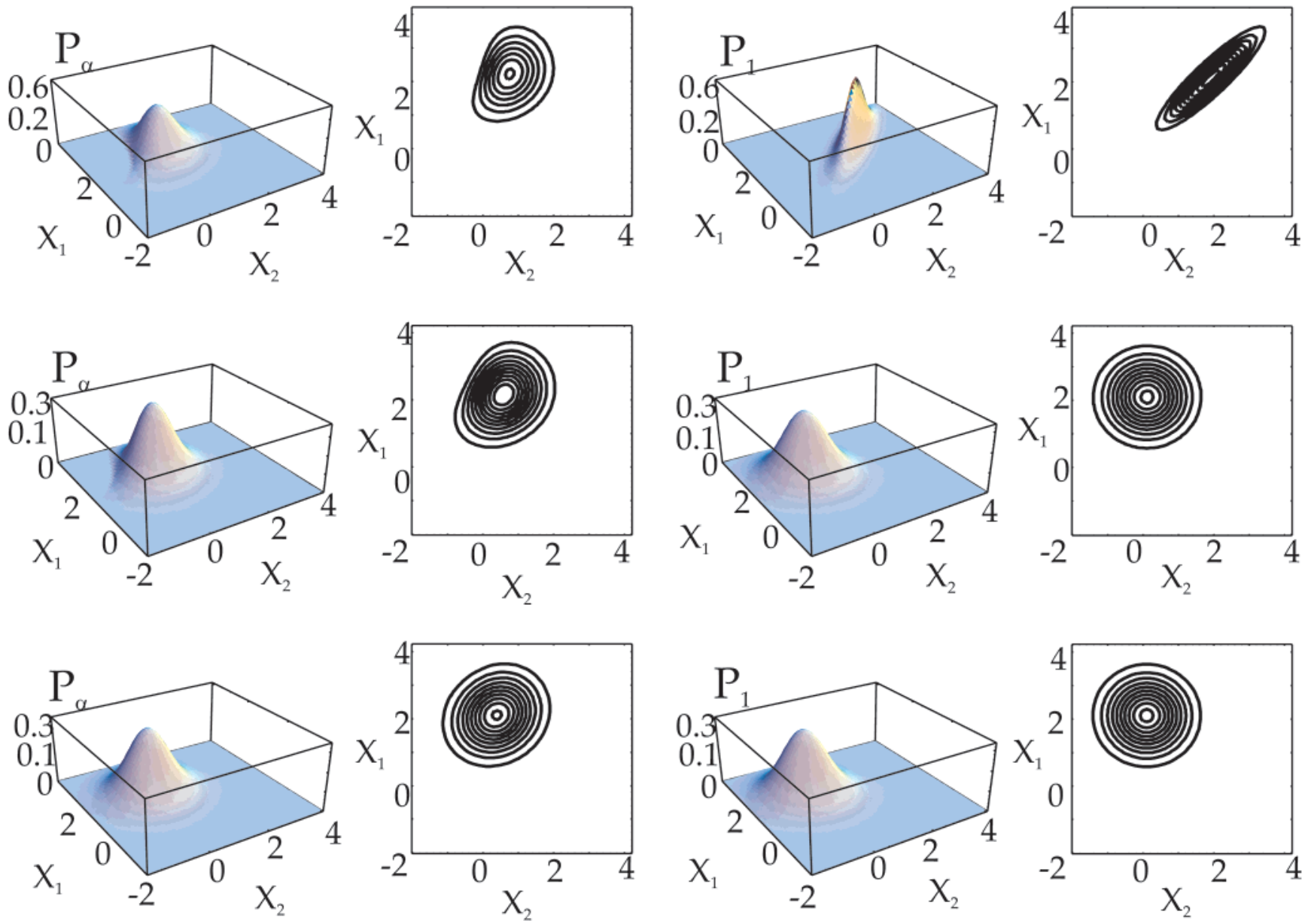

Figure 3. The joint probability $P_{\alpha}\left(X_{2}, \bar{t}_{2} ; X_{1}, \bar{t}_{1}\right)$ vs $X_{1}$ and $X_{2}$ (̊) for models M3 (left) and M4 (right) for $\bar{t}_{1}=50.0 \mathrm{ps}$ and $\bar{t}_{2}=1.0 \mathrm{ps}$ (top), $\bar{t}_{1}=$ $50.0 \mathrm{ps}$ and $\bar{t}_{2}=200 \mathrm{ps}$ (middle), and $\bar{t}_{1}=50.0 \mathrm{ps}$ and $\bar{t}_{2}=1.5 \mathrm{~ns}$ (bottom). Contour plots are shown to the right of $2 \mathrm{D}$ surfaces.

Inserting eq 10 and the scaled potential $\tilde{V}(X)$ in the right-hand side of eq 18 , we obtain

$$
\begin{aligned}
& W_{\alpha}\left(X_{2}, t_{2} ; X_{1}, t_{1}\right)= \\
& \frac{1}{\sqrt{2 \pi \theta^{n}=0} 2^{n} n !} \sum_{\alpha}^{\infty} \frac{1}{}\left(-n \tilde{t}_{20}^{\alpha}\right) E_{\alpha}\left(n \tilde{t}_{10}^{\alpha}\right) H_{n}\left(\tilde{X}_{2} / \sqrt{2}\right) H_{n}\left(\tilde{X}_{1} / \sqrt{2}\right) \mathrm{e}^{-\tilde{X}_{2}^{2} / 2}
\end{aligned}
$$

For $\alpha=1$, we get

$$
\begin{aligned}
W_{\alpha=1}\left(X_{2}, t_{2} ; X_{1}, t_{1}\right) & \\
= & \mathrm{e}^{\tilde{V}\left(X_{1}\right) / 2-\tilde{V}\left(X_{2}\right) / 2} \sum_{n=0}^{\infty} \psi_{n}\left(X_{2}\right) \psi_{n}\left(X_{1}\right) \mathrm{e}^{-\lambda_{n, 1} t_{21}} \\
& =\frac{1}{\sqrt{2 \pi} \theta^{n=0} 2^{n} n !} \frac{1}{\mathrm{e}^{-n \lambda_{1} t_{21}}} H_{n}\left(\tilde{X}_{2} / \sqrt{2}\right) H_{n}\left(\tilde{X}_{1} / \sqrt{2}\right) \mathrm{e}^{-\tilde{X}_{2}^{2} / 2}
\end{aligned}
$$

By use of the summation formula for the Hermite polynomials, ${ }^{37,38}$ it can be brought to a form of eq 16. Note that for $\alpha=$ $1 W_{\alpha}$ represents a stationary process and it only depends on $t_{21}$. For $\alpha<1$, the process is nonstationary and $W_{\alpha}$ depends on both $t_{20}$ and $t_{10}$.
In the limit $t_{21}=t_{2}-t_{1} \rightarrow \infty$, we recover the equilibrium Boltzmann distribution, that is,

$$
\begin{aligned}
\rho_{\mathrm{eq}}\left(X_{2}\right) & \equiv \lim _{\Delta t \rightarrow \infty} W_{\alpha}\left(X_{2}, t_{2} ; X_{1}, t_{1}\right) \\
& =\mathrm{e}^{\tilde{V}\left(X_{1}\right) / 2-\tilde{V}\left(X_{2}\right) / 2} \\
& \times \lim _{\Delta t \rightarrow \infty} \sum_{n=0}^{\infty} \psi_{n}\left(X_{2}\right) \psi_{n}\left(X_{1}\right) E_{\alpha}\left(-\lambda_{n, \alpha} t_{20}^{\alpha}\right) E_{\alpha}\left(\lambda_{n, \alpha} t_{10}^{\alpha}\right) \\
& =\mathrm{e}^{\tilde{V}\left(X_{1}\right) / 2-\tilde{V}\left(X_{2}\right) / 2} \psi_{0}\left(X_{2}\right) \psi_{0}\left(X_{1}\right) \\
& =[1 /(2 \pi \theta)]^{1 / 2} \mathrm{e}^{-X_{2}^{2} /(2 \theta)}
\end{aligned}
$$

where $\psi_{0}(X)$ is the minimum uncertainty coherent state of the harmonic oscillator, given by $\psi_{0}(X)=[1 /(2 \pi \theta)]^{1 / 4}$ $\exp \left[-\tilde{X}^{2} / 2\right]$. Equations 19 and 20 will be used in the following calculations.

\section{Conditional and Joint Probabilities}

To study the fluctuation statistics, we have computed the twopoint conditional distribution of $X$ for models M1 and M2, and the three-point joint distribution of $X$ for models M3 and M4. The parameters of these models are summarized in Table 1. Models M1 and M3 correspond to subdiffusion $(\alpha=0.5)$, and models M2 and M4 represent ordinary Brownian diffusion ( $\alpha$ $=1)$. We set the diffusion and friction constants for $\alpha=0.5$ and $\alpha=1$ to be equal, that is, $K_{1}=K_{\alpha}$ and $\eta_{\alpha}=\eta_{1}$. In models 


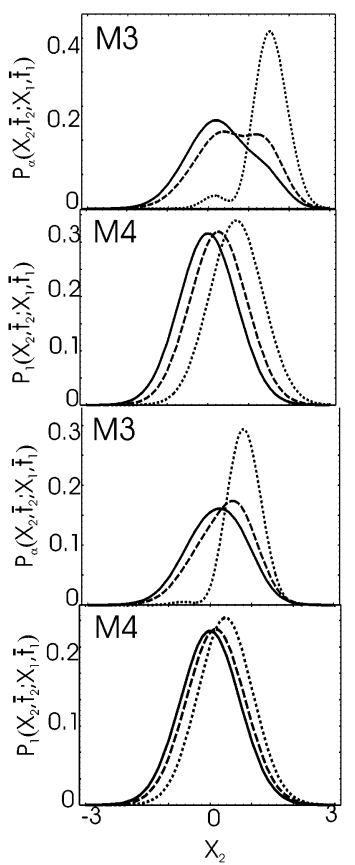

Figure 4. Constant $\bar{t}_{2}$ sections of $P_{\alpha}\left(X_{2}, \bar{t}_{2} ; X_{1}, \bar{t}_{1}\right)$ vs $X_{2}(\AA)$ for $X_{1}=$ $2.0 \AA$ (upper panels) and $X_{1}=1.0 \AA$ (lower panels) for models M3 and M4. For model M3, $\bar{t}_{2}=8.0 \mathrm{ps}(\cdots), 200 \mathrm{ps}(---)$, and $1.5 \mathrm{~ns}$ $(-)$; for model M4, $\bar{t}_{2}=8.0 \mathrm{ps}(\cdots), 20.0 \mathrm{ps}(---)$ and $1.0 \mathrm{~ns}(-)$.

TABLE 1: Parameters for Models M1-M4 Used in Calculation of the Two-Point Conditional and Three-Point Joint Distribution of Donor-Acceptor Distance in Figures 1-4

\begin{tabular}{cccccc}
\hline model & $K_{1} \times 10^{2}\left(\mathrm{~s}^{-1}\right)$ & $\eta_{1}\left(\mathrm{~s}^{-1}\right)$ & $\alpha$ & $x_{0}(\AA)$ & $t_{0}(\mathrm{ps})$ \\
\hline M1 & 5.0 & 10.0 & 0.5 & & \\
M2 & 5.0 & 10.0 & 1.0 & & \\
M3 & 5.0 & 10.0 & 0.5 & 2.0 & 0 \\
M4 & 5.0 & 10.0 & 1.0 & 2.0 & 0
\end{tabular}

TABLE 2: Parameters for Models M5-M8 Used in Calculation of $C_{\alpha}^{\mathrm{FRET}}\left(\bar{t}_{2}, \bar{t}_{1}\right)$ in Figure 5

\begin{tabular}{cccccccc}
\hline model & $K_{1} \times 10^{2}\left(\mathrm{~s}^{-1}\right)$ & $\eta_{1}\left(\mathrm{~s}^{-1}\right)$ & $\alpha$ & $R_{0}(\AA)$ & $\gamma_{0}\left(\mathrm{~s}^{-1}\right)$ & $x_{0}(\AA)$ & $t_{0}(\mathrm{ps})$ \\
\hline M5 & 5.0 & 10.0 & 0.5 & 10.0 & 1.0 & 2.0 & 0 \\
M6 & 5.0 & 10.0 & 1.0 & 10.0 & 1.0 & 2.0 & 0 \\
M7 & 5.0 & 10.0 & 0.5 & 10.0 & 1.0 & 2.0 & 0 \\
M8 & 5.0 & 10.0 & 1.0 & 10.0 & 1.0 & 2.0 & 0
\end{tabular}

TABLE 3: Parameters for Models M9-M12 Used in Calculation of $C_{\alpha}^{\mathrm{ET}}\left(\bar{t}_{2}, \bar{t}_{1}\right)$ in Figures 6

\begin{tabular}{lccccccc}
\hline model & $K_{1} \times 10^{2}\left(\mathrm{~s}^{-1}\right)$ & $\eta_{1}\left(\mathrm{~s}^{-1}\right)$ & $\alpha$ & $k_{0}\left(\mathrm{~s}^{-1}\right)$ & $\beta(1 / \AA)$ & $x_{0}(\AA)$ & $t_{0}(\mathrm{ps})$ \\
\hline M9 & 5.0 & 10.0 & 0.5 & 10.0 & 2.0 & 2.0 & 0 \\
M10 & 5.0 & 10.0 & 1.0 & 10.0 & 2.0 & 2.0 & 0 \\
M11 & 5.0 & 10.0 & 0.5 & 10.0 & 2.0 & 2.0 & 0 \\
M12 & 5.0 & 10.0 & 1.0 & 10.0 & 2.0 & 2.0 & 0
\end{tabular}

M3 and M4, $X$ starts off from the nonequilibrium value $X_{0}=$ $x_{0} \neq 0$ at time $t_{0}=0$.

Note that for $\alpha=0.5$ the Mittag-Leffler function defined in eq 13 reduces to $\mathrm{e}^{n^{2} \tilde{t}} \operatorname{erfc}(n \sqrt{\tilde{t}})$. This was used to compute both conditional and joint probabilities for $\alpha=0.5$ utilizing series expansion given by eq 19. The series was truncated at $n$ $=60$.

Using $W_{\alpha}$ (eqs 19 and 20), in Figure 1 we compare the _equilibrium-weighted conditional probability $W_{\alpha}\left(X_{2}, \bar{t}_{2} ; X_{1}, \bar{t}_{1}\right) \rho_{\mathrm{eq}}\left(X_{1}\right)$ vs $X_{1}$ and $X_{2}$ for a fixed time difference $\bar{t}_{1}=t_{1}=1 \mathrm{ps}$ and a series of $\bar{t}_{2}=t_{2}-t_{1}=1,10$, and $100 \mathrm{ps}$ for models M1 and M2 keeping $K_{\alpha}$ and $\eta_{1}$ fixed. $\rho_{\text {eq }}(X)$ is given by eq 21 .
Initial correlations between $X_{1}$ and $X_{2}$ are reflected in the enhanced $X_{1}=X_{2}$ diagonal feature. This feature gradually vanishes for longer $\bar{t}_{2}$ as the conditional probability becomes a two-dimensional Gaussian symmetrically distributed with respect to $X_{1}$ and $X_{2}$. However, as seen from the contour plots for longer $\bar{t}_{2}$, the variation of probability in $X_{1}, X_{2}$ space is considerably slower for model M1. This reflects longer lasting $X_{1}, X_{2}$ correlations for subdiffusion compared with ordinary Brownian motion. Because Brownian diffusion is a Markovian process, $W_{\alpha=1}$ depends only on the difference $t_{2}-t_{1}$. Subdiffusive dynamics is in contrast intrinsically non-Markovian, and $W_{\alpha=0.5}$ depends on both $t_{1}$ and $t_{2}$.

Using eq 19, we have computed the joint probability to be in $X_{2}$ at time $t_{2}$ given that it was in $X_{1}$ at time $t_{1}<t_{2}$ and in $X_{0}=x_{0}$ at time $t_{0}=0$,

$$
\begin{aligned}
& P_{\alpha}\left(X_{2}, t_{2} ; X_{1}, t_{1} ; x_{0}, t_{0}\right) \\
& \equiv \int_{-\infty}^{\infty} \mathrm{d} X_{0} W_{\alpha}\left(X_{2}, t_{2} ; X_{1}, t_{1}\right) W_{\alpha}\left(X_{1}, t_{1} ; X_{0}, t_{0}\right) \rho_{\mathrm{eq}}\left(X_{0}\right) \\
& =\frac{1}{2 \pi \theta} \mathrm{e}^{-\left(X_{1}^{2}+X_{2}^{2}\right) /(2 \theta)} \\
& \times \sum_{m=0}^{\infty} \frac{1}{m !} E_{\alpha}\left(-m \bar{t}_{1}^{\alpha}\right) H_{m}\left(\tilde{X}_{1} / \sqrt{2}\right)\left(\tilde{x}_{0} / \sqrt{2}\right)^{m} \\
& \times \sum_{n=02^{n} n !}^{\infty} \frac{1}{\alpha} E_{\alpha}\left(-n \tilde{t}_{2}^{\alpha}\right) E_{\alpha}\left(-n \tilde{t}_{1}^{\alpha}\right) H_{n}\left(\tilde{X}_{2} / \sqrt{2}\right) H_{n}\left(\tilde{X}_{1} / \sqrt{2}\right)
\end{aligned}
$$

Figure 2 displays $P_{\alpha}\left(X_{2}, \bar{t}_{2} ; X_{1}, \bar{t}_{1} ; x_{0}, 0\right)$ vs $X_{1}$ and $X_{2}$ for fixed $\bar{t}_{1}=1 \mathrm{ps}$ and a series of $\bar{t}_{2}=1 \mathrm{ps}, 200 \mathrm{ps}$, and $1.5 \mathrm{~ns}$ assuming that $X_{0}$ is Gaussian-distributed around $\tilde{x}_{0}=2 \AA$ at time $t_{0}=0$ for models M3 and M4. The $\alpha=0.5$ dynamics differs qualitatively from its Brownian counterpart $\alpha=1$. Although for both models the probability undergoes diffusion in $X_{1}, X_{2}$ space with a finite shift of the most probable configuration $\left(X_{1}^{\max }, X_{2}^{\max }\right), P_{\alpha=0.5}$ is separated into two peaks while retaining correlations between $X_{1}$ and $X_{2}$ even for $\bar{t}_{2}=1.5 \mathrm{~ns}$. These correlations disappear for $P_{\alpha=1}$ already at $\bar{t}_{2}=200 \mathrm{ps}$, where it assumes a two-dimensional $X_{1}, X_{2}$-symmetric Gaussian form.

In Figure 3, we repeated these calculations for $\bar{t}_{1}=50$ ps. $P_{\alpha=0.5}$ remains asymmetric even at $\bar{t}_{2}=200 \mathrm{ps}$ and $1.5 \mathrm{~ns}$ implying $X_{1}, X_{2}$ correlations, whereas $P_{\alpha=1}$ is Gaussian. Comparing contour plots of $P_{\alpha=0.5}$ and $P_{\alpha=1.0}$ for short $\bar{t}_{1}$ and $\bar{t}_{2}$ (top panels in Figures 2 and 3) with long $\bar{t}_{1}$ and $\bar{t}_{2}$, we see that a decay of $X_{1}, X_{2}$ correlations is faster for subdiffusion for short times but slower for long times compared with $\alpha=1$. This can be understood because the Mittag-Leffler function interpolates between a faster decaying stretched exponential at short times and a power law at long times, compared with the exponential function.

In Figure 4 , we display $P_{\alpha}\left(X_{2}, \bar{t}_{2} ; X_{1}, \bar{t}_{1}\right)$ vs $X_{2}$ and $\bar{t}_{2}$ for fixed $X_{1}=2.0$ and $1.0 \AA$ and $\bar{t}_{1}=1$ ps and constant $\bar{t}_{2}$ sections of $P_{\alpha=0.5}$ for $\bar{t}_{2}=8 \mathrm{ps}, 200 \mathrm{ps}$, and $1.5 \mathrm{~ns}$ and $P_{\alpha=1.0}$ for $\bar{t}_{2}=8 \mathrm{ps}$, $20 \mathrm{ps}$, and $1.0 \mathrm{~ns}$ for models M3 and M4, respectively. Here again, we find a notable difference between $\alpha=0.5$ and $\alpha=$ 1. $P_{\alpha=1.0}$ diffuses gradually to equilibrium $X_{2}=0$ retaining a fixed Gaussian $\bar{t}_{2}$ profile, whereas $P_{\alpha=0.5}$ builds up its density around $X_{2}=0$ almost immediately by funneling the distribution from $X_{1}$. Comparing the magnitude of constant $\bar{t}_{2}$ sections of $P_{\alpha=0.5}$ and $P_{\alpha=1}$, we see that as $X_{2}$ relaxes to zero with increasing 

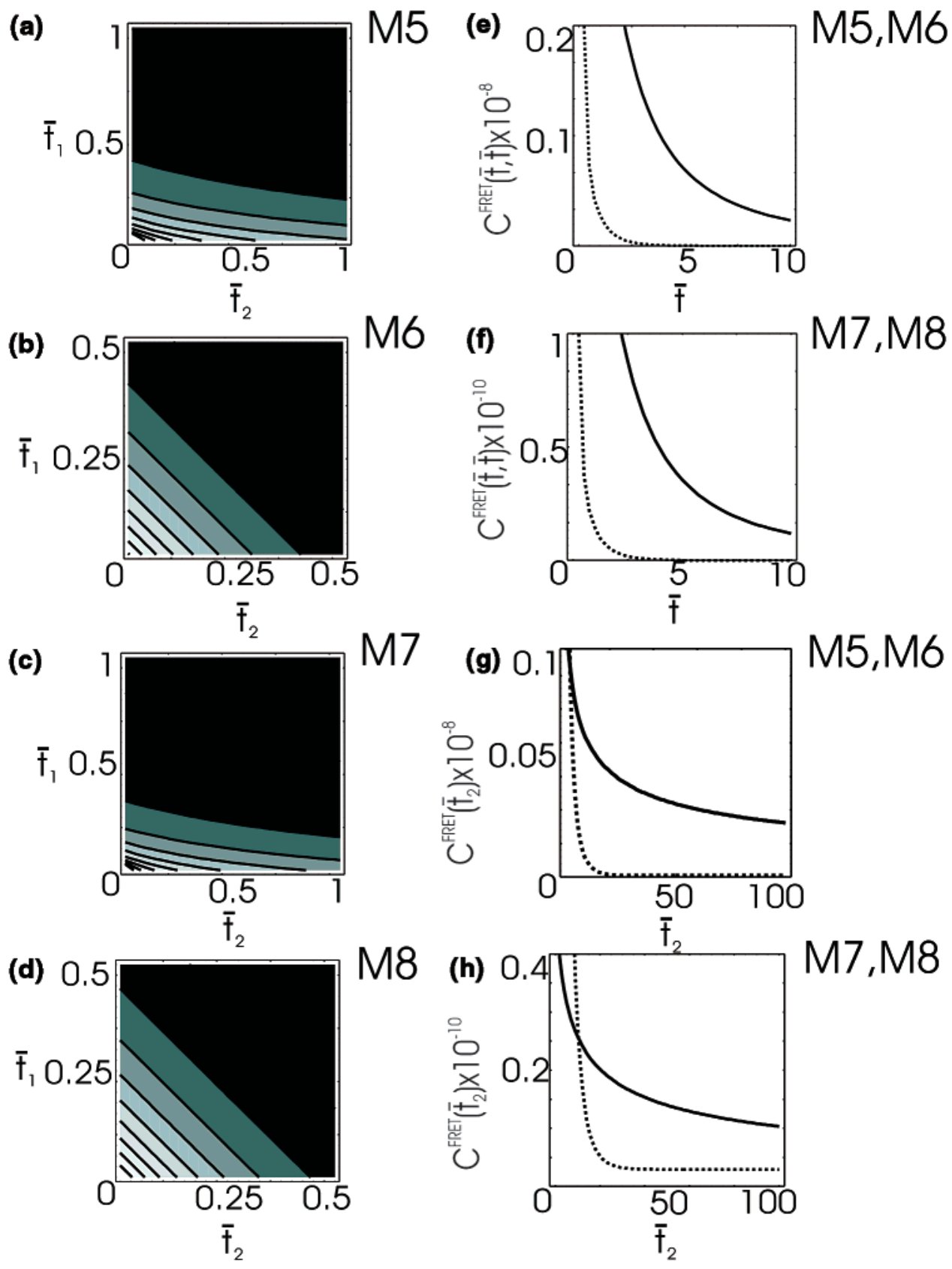

Figure 5. $C_{\alpha}^{\mathrm{FRET}}\left(\bar{t}_{2}, \bar{t}_{1}\right)\left(\mathrm{ps}^{3}\right)$ vs $\bar{t}_{1}$ and $\bar{t}_{2}(\mathrm{ps})$ for models (a) M5, (b) M6, (c) M7, and (d) M8, the diagonal section $C_{\alpha}^{\mathrm{FRET}}(\bar{t}, \bar{t})\left(\mathrm{ps}^{3}\right)$ vs $\bar{t}_{1}=\bar{t}_{2}=\bar{t}$ (ps) for models (e) M5 and M6 and (f) M7 and M8, and constant $\bar{t}_{1}=1$ ps sections $C_{\alpha}^{\text {FRET }}\left(\bar{t}_{2}\right)\left(\mathrm{ps}^{3}\right)$ vs $\bar{t}_{2}$ (ps) for models (g) M5 and M6 and (h) M7 and M8. Models M5 and M7 (M6 and M8) are denoted by solid (dotted) lines.

$\bar{t}_{2}, P_{\alpha}$ becomes equilibrium distributed for both $\alpha=0.5$ and $\alpha=1.0$. However, $P_{\alpha=0.5}$ shows a residual density positioned around the original $X_{1}$ even for large $\bar{t}_{2}$.

Note that because in our calculations of $W_{\alpha}\left(X_{2}, \bar{t}_{2} ; a X_{1}, \bar{t}_{1}\right) \rho_{\mathrm{eq}}{ }^{-}$ $\left(X_{1}\right)$ and $P_{\alpha}\left(X_{2}, \bar{t}_{2} ; X_{1}, \bar{t}_{1}\right)$ for $\alpha=0.5$ we used a truncated series expansion (eq 19), these quantities do not exhibit nondifferentiable cusps (see discussion in section 5.4 of ref 31).

\section{Multitime Correlation Function of Fluorescence Lifetime}

The Green function, $W_{\alpha}\left(X_{2}, t_{2} ; X_{1}, t_{1}\right)$, may be used to compute $n$-point correlation functions of arbitrary variables that depend on $X(t)$. In this section, we compute the three-time correlation functions of the fluorescence lifetime defined by

$$
\begin{aligned}
& \left\langle\tau\left(t_{2}\right) \tau\left(t_{1}\right) \tau\left(t_{0}\right)\right\rangle \\
& \quad \equiv \int_{-\infty}^{\infty} \int_{-\infty}^{\infty} \int_{-\infty}^{\infty} \mathrm{d} X_{0} \mathrm{~d} X_{1} \mathrm{~d} X_{2} \tau\left(X_{0}\right) \tau\left(X_{1}\right) \tau\left(X_{2}\right) \\
& \quad \times W_{\alpha}\left(X_{2}, t_{2} ; X_{1}, t_{1}\right) W_{\alpha}\left(X_{1}, t_{1} ; X_{0}, t_{0}\right) \rho_{\text {eq }}\left(X_{0}\right)
\end{aligned}
$$

$\left\langle\tau\left(t_{2}\right) \tau\left(t_{1}\right) \tau\left(t_{0}\right)\right\rangle$ is computed by substituting eq 19 for $\alpha=0.5$ and eq 20 for $\alpha=1$ and eq 1 for FRET and eq 2 for ET into eq 23. The resulting expression for $C_{\alpha}^{\mathrm{FRET}}\left(t_{2}, t_{1}\right)$ is given by eq $\mathrm{B} 2$ and $C_{\alpha}^{\mathrm{ET}}\left(t_{2}, t_{1}\right)$ is given by eq B7.

We have computed $\left\langle\tau\left(t_{2}\right) \tau\left(t_{1}\right) \tau\left(t_{0}\right)\right\rangle$ for eight models: models M5-M8 are for FRET and models M9-M12 are for ET. In these models, we varied the diffusion coefficient $K_{\alpha}$. $\left\langle\tau\left(t_{2}\right) \tau\left(t_{1}\right) \tau\left(t_{0}\right)\right\rangle$ for models M5, M7, M9, and M11 of subdiffusion $(\alpha=0.5)$ is compared with the same quantity computed 

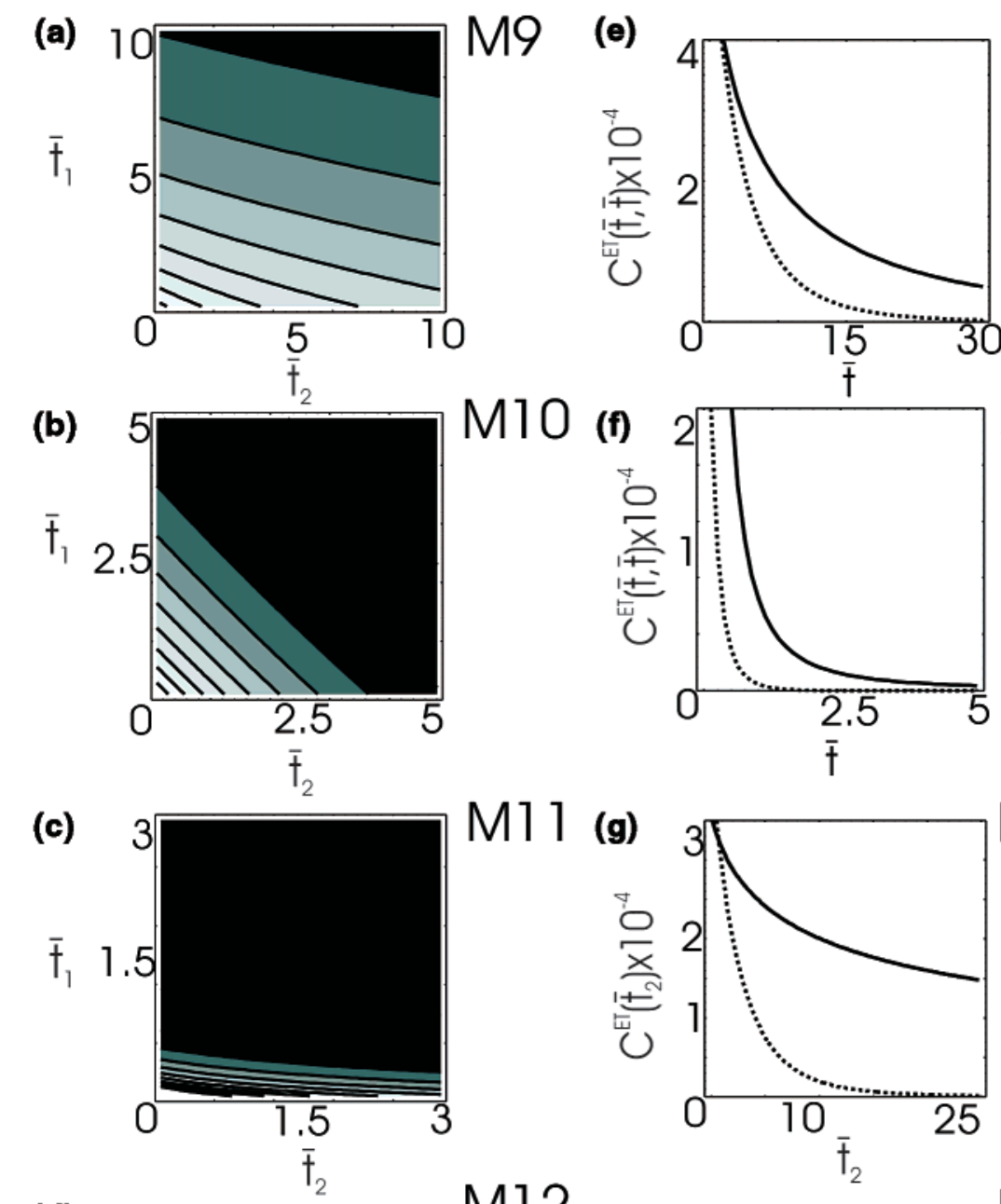

$\mathrm{M} 11, \mathrm{M} 12$
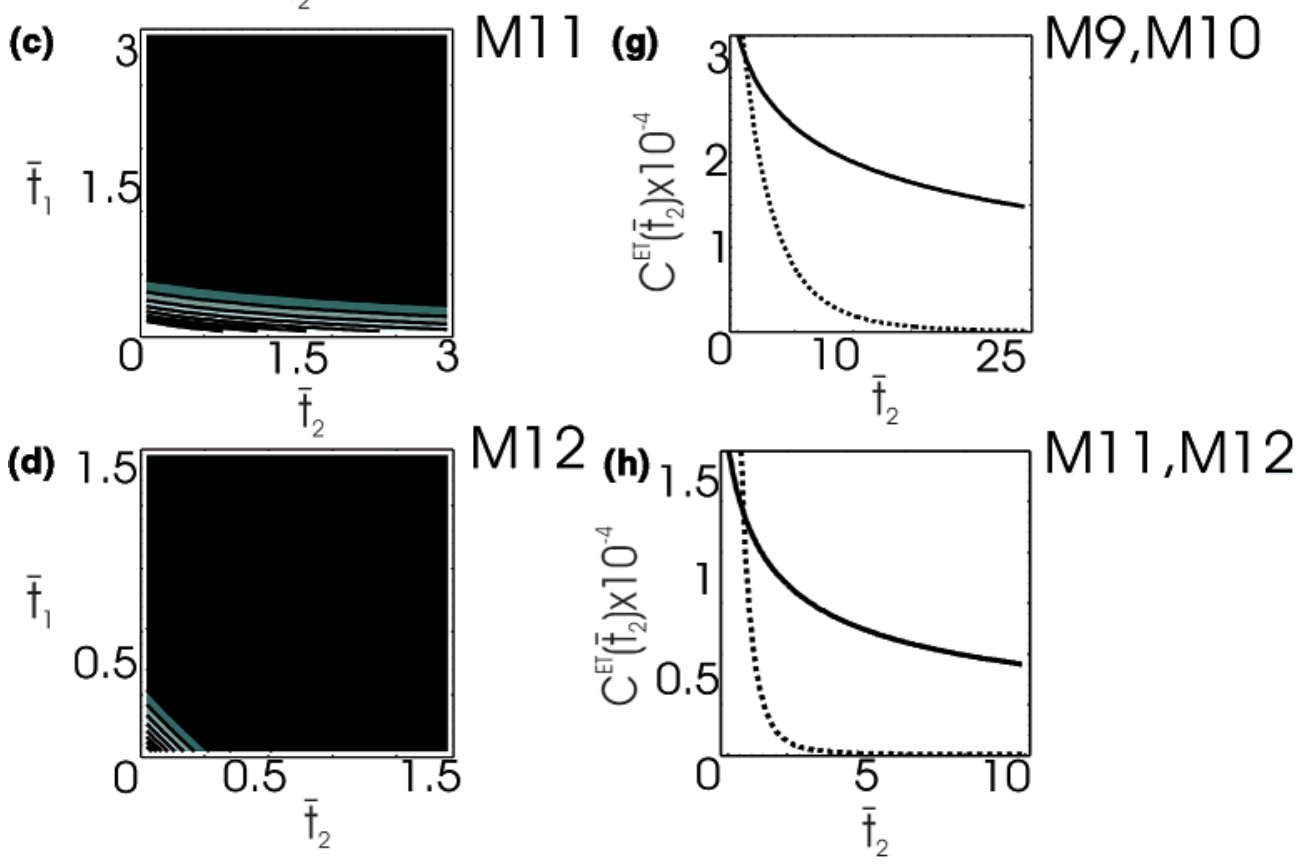

Figure 6. $\left.C_{\alpha}^{\mathrm{ET}} \bar{t}_{2}, \bar{t}_{1}\right)\left(\mathrm{ps}^{3}\right)$ vs $\bar{t}_{1}$ and $\bar{t}_{2}$ (ps) for models (a) M9, (b) M10, (c) M11, and (d) M12, the diagonal section $\left.C_{\alpha}^{\mathrm{ET}} \bar{t}, \bar{t}\right)\left(\mathrm{ps}^{3}\right) \mathrm{vs} \bar{t}_{1}=\bar{t}_{2}=\bar{t}$ (ps) for models (e) M9 and M10 and (f) M11 and M12, and constant $\bar{t}_{1}=1$ ps sections $\left.C_{\alpha}^{\mathrm{ET}} \bar{t}_{2}\right)\left(\mathrm{ps}{ }^{3}\right)$ vs $\bar{t}_{2}$ (ps) for models (g) M9 and M10 and (h) M11 and M12. Models M9 and M11 (M10 and M12) are denoted by solid (dotted) lines.

for Brownian diffusion models M6, M8, M10, and M12. We set the diffusion and friction constants for models with $\alpha=$ 0.5 and $\alpha=1$ to be equal, that is, $K_{1}=K_{\alpha}$ and $\eta_{\alpha}=\eta_{1}$, and assume a near-equilibrium initial condition for $X$, that is, $X_{0}=$ $2 \AA$ at time $t_{0}=0$. In FRET models, we set $R_{0}=10.0 \AA$ and $\gamma_{0}=1.0 \mathrm{~s}^{-1}$. In ET models, we use $k_{0}=10 \mathrm{~s}^{-1}$ and $\beta=2.0$ $\AA^{-1}$. In FRET models M5 and M6 (M7 and M8), $K_{1}=\eta_{1}\left(K_{1}\right.$ $\left.>\eta_{1}\right)$. In ET models M9 and M10 (M11 and M12), $K_{1}<\eta_{1}$ $\left(K_{1}>\eta_{1}\right)$. Parameters used in FRET and ET models are summarized in Tables 2 and 3, respectively.

Figure 5 compares $C_{\alpha}^{\mathrm{FRET}}\left(\bar{t}_{2}, \bar{t}_{1}\right)$ vs $\bar{t}_{1}$ and $\bar{t}_{2}$ for models M5-M8 (where we varied the magnitude of $\left.K_{1}\right) \cdot C_{\alpha}^{\mathrm{FRET}}\left(\bar{t}_{2}, \bar{t}_{1}\right)$ is weaker and undergoes a faster decay for larger values of $K_{1}$ for both subdiffusion and Brownian diffusion. To highlight this point, we also display diagonal $\bar{t}_{1}=\bar{t}_{2}$ sections of $C_{\alpha}^{\text {FRET }}$ for models M5-M8 and constant $\bar{t}_{1}=1$ ps sections of $C_{\alpha}^{\text {FRET }}\left(\bar{t}_{2}, \bar{t}_{1}\right)$. Slowly decaying correlations are clearly observed for $\alpha=0.5$.

The plots show a qualitative difference in the fluorescence lifetime correlations. Because of the absence of memory in $X$ for $\alpha=1$, the decay of $C_{\alpha=1}^{\mathrm{FRET}}\left(\bar{t}_{2}, \bar{t}_{1}\right)$ with $\bar{t}_{1}$ and $\bar{t}_{2}$ occurs on the same time scale and contour plots of $C_{\alpha=1}$ are symmetric with respect to interchange $\bar{t}_{1} \leftrightarrow \bar{t}_{2}$ for $\alpha=1.0$ models. However, because of non-Markovian evolution of $X$, this is not the case for $C_{\alpha=0.5}^{\mathrm{FRET}}\left(\bar{t}_{2}, \bar{t}_{1}\right) \quad(\alpha=0.5)$, which_shows longer lasting correlations among $\bar{t}_{2}$ compared with $\bar{t}_{1}$.

As discussed earlier, the non-Markovian subdiffusive dynamics follows a faster decay for short $\left(\bar{t} \ll 1 / \lambda_{1}\right.$, stretched exponential behavior) and slower decay at long $\left(\bar{t} \gg 1 / \lambda_{1}\right.$, a power law) time intervals compared with the exponential decay 
in case of the Markovian dynamics. Because of this, contours of $C_{\alpha=0.5}^{\mathrm{FRET}}\left(\bar{t}_{2}, \bar{t}_{1}\right)$ are intrinsically asymmetric and are more stretched along $\bar{t}_{2}$. This pattern persists as $K_{1} / \eta_{1}$ is varied.

$C_{\alpha}^{\mathrm{ET}}\left(\bar{t}_{2}, \bar{t}_{1}\right)$ for models M9-M12 is shown in Figure 6 (where we varied the magnitude of $\left.K_{1}\right)$. We also show diagonal $\bar{t}_{1}=\bar{t}_{2}$ sections and constant $\bar{t}_{1}=1$ ps profiles of $C_{\alpha}^{\mathrm{ET}}\left(\bar{t}_{2}, \bar{t}_{1}\right)$ for models M9-M12. Similar to FRET, $C_{\alpha}^{\mathrm{ET}}\left(\bar{t}_{2}, \bar{t}_{1}\right)$ has smaller amplitude and follows a faster decay as $K_{1}$ is increased for both $\alpha=0.5$ and $\alpha=1$, and the interdye distance correlations live longer for $\alpha=0.5$. Because of the absence (presence) of memory in Brownian diffusion (subdiffusion) models, contour plots are symmetric (asymmetric) with respect to $\bar{t}_{1} \leftrightarrow \bar{t}_{2}$ for $C_{\alpha=1.0}^{\mathrm{ET}}\left(C_{-\alpha=0.5}^{\mathrm{ET}}\right)$. Comparison of contour plots, diagonal sections $C_{\alpha}(t, t)$, and fixed $\bar{t}_{1}=1$ ps profiles $C_{\alpha}\left(\bar{t}_{2}\right)$ for FRET and ET shows that because in FRET the fluorescence quenching rate varies with $X$ as $\sim X^{-6}$ and thus follows a slower (power-law) decay compared to exponential decay rate for ET, $C_{\alpha}^{\text {FRET }}$ retains correlations of lifetimes for longer times compared to $C_{\alpha}^{\mathrm{ET}}$.

Acknowledgment. The support of the Division of Chemical Sciences, Office of Basic Energy Sciences, U.S. Department of Energy, Grant DE-FG02-01ER 15155, is gratefully acknowledged. We thank Prof. Sunney Xie for sharing ref 17 prior to publication and Profs. J. Klafter and R. Metzler for useful discussions.

\section{Appendix A: Backward Propagator for the FFPE}

The backward propagator is the solution of the backward FFPE,

$$
\frac{\mathrm{d}}{\mathrm{d} t_{1}} W_{\alpha}\left(X_{2}, t_{2} ; X_{1}, t_{1}\right)=-{ }_{0} D_{\mathrm{t}_{1}}{ }^{1-\alpha} L_{\mathrm{FP}}^{\dagger}\left(X_{1}\right) W_{\alpha}\left(X_{2}, t_{2} ; X_{1}, t_{1}\right)
$$

where the backward Fokker-Planck operator is $L_{\mathrm{FP}}^{\dagger}$, defined by $^{31}$

$$
L_{\mathrm{FP}}^{\dagger}=-\frac{V^{\prime}(X)}{m \eta_{\alpha}} \frac{\partial}{\partial X}+K_{\alpha} \frac{\partial^{2}}{\partial X^{2}}
$$

Because ${ }_{0} D_{t}{ }^{1-\alpha} L_{\mathrm{FP}}$ and ${ }_{0} D_{t}{ }^{1-\alpha} L_{\mathrm{FP}}^{\dagger}$ are not Hermitian conjugates, to obtain the eigenfunction expansion for the backward propagator, we need the adjoint of operator $\exp [\bar{V}(X)]_{0} D_{t}{ }^{1-\alpha} L_{\mathrm{FP}}$. It is easy to see that

$$
\begin{aligned}
{\left[\mathrm{e}^{\tilde{V}}(X){ }_{0} D_{t}{ }^{1-\alpha} L_{\mathrm{FP}}(X)\right]^{\dagger} } & \equiv\left[{ }_{0} D_{t}{ }^{1-\alpha} L_{\mathrm{FP}}(X)\right]^{\dagger} \mathrm{e}^{\tilde{V}(X)} \\
& =\left[\frac{1}{\Gamma(\alpha)} \frac{\partial}{\partial t} \int_{0}^{t} \mathrm{~d} t^{\prime} \frac{L_{\mathrm{FP}}^{\dagger}(X)}{\left(t-t^{\prime}\right)^{1-\alpha}}\right] \mathrm{e}^{\tilde{V}(X)} \\
& ={ }_{0} D_{t}{ }^{1-\alpha} L_{\mathrm{FP}}^{\dagger}(X) \mathrm{e}^{\tilde{V}(X)} \\
& ={ }_{0} D_{t}{ }^{1-\alpha} \mathrm{e}^{\tilde{V}(X)} L_{\mathrm{FP}}(X)
\end{aligned}
$$

where in the last line of eq A 3 we used the following property, ${ }^{37}$

$$
\left[\mathrm{e}^{\tilde{V}(X)} L_{\mathrm{FP}}(X)\right]^{\dagger} \equiv L_{\mathrm{FP}}^{\dagger}(X) \mathrm{e}^{\tilde{V}(X)}=\mathrm{e}^{\tilde{V}(X)} L_{\mathrm{FP}}(X)
$$

The operators $\exp [\tilde{V}(X)] L_{\mathrm{FP}}(X)$ and

$$
L(X)=\mathrm{e}^{\tilde{V}(X) / 2} L_{\mathrm{FP}}(X) \mathrm{e}^{-\tilde{V}(X) / 2}=\mathrm{e}^{-\tilde{V}(X) / 2} L_{\mathrm{FP}}^{\dagger}(X) \mathrm{e}^{\tilde{V}(X) / 2}
$$

are now Hermitian. Equation A3 implies that the fractional operators $\mathrm{e}^{\tilde{V}(X)}{ }_{0} D_{t}{ }^{1-\alpha} L_{\mathrm{FP}}(X)$ and ${ }_{0} D_{t}{ }^{1-\alpha} L_{F P}^{\dagger}(X) \mathrm{e}^{\tilde{V}(X)}$ have the same eigenvalues $\lambda_{n, \alpha}$ and orthonormal basis set $\left\{\psi_{n}(X)\right\}$ (see eq 11). This allows us to expand the backward propagator ${ }_{0} D_{t}^{1-\alpha} L_{F P}^{\dagger}(X)(t \leq 0)$ as

$$
\begin{aligned}
& W_{\alpha}^{-}\left(X_{1}, t_{1} ; X_{0}, t_{0}=0\right) \\
& =E_{\alpha}\left(L_{\mathrm{FP}}^{\dagger}\left(X_{0}\right) t_{1}^{\alpha}\right) \delta\left(X_{1}-X_{0}\right) \\
& =\mathrm{e}^{\tilde{V}\left(X_{1}\right)} \sum_{n=0}^{\infty} E_{\alpha}\left(L_{\mathrm{FP}}^{\dagger}\left(X_{0}\right) t_{1}^{\alpha}\right) \phi_{n}\left(X_{1}\right) \phi_{n}\left(X_{0}\right) \\
& =\mathrm{e}^{\tilde{V}\left(X_{1}\right)} \sum_{n=0}^{\infty} \phi_{n}\left(X_{1}\right) \phi_{n}\left(X_{0}\right) E_{\alpha}\left(-\lambda_{n, \alpha} t_{1}^{\alpha}\right) \\
& =\mathrm{e}^{\tilde{V}\left(X_{0}\right) / 2-\tilde{V}\left(X_{1}\right) / 2} \sum_{n=0}^{\infty} \psi_{n}\left(X_{1}\right) \psi_{n}\left(X_{0}\right) E_{\alpha}\left(-\lambda_{n, \alpha} t_{1}^{\alpha}\right)
\end{aligned}
$$

The propagator between arbitrary times $t_{1}$ and $t_{2}$ can now be obtained by substituting eqs 12 and A6 into eq 17, that is,

$$
\begin{aligned}
& W_{\alpha}\left(X_{2}, t_{2} ; X_{1}, t_{1}\right) \\
&=\int_{-\infty}^{\infty} \mathrm{d} X_{0} W_{\alpha}^{+}\left(X_{2}, t_{2} ; X_{0}, 0\right) W_{\alpha}^{-}\left(X_{0}, 0 ; X_{1}, t_{1}\right) \\
&=\int_{-\infty}^{\infty} \mathrm{d} X_{0} \mathrm{e}^{\tilde{V}\left(X_{0}\right) / 2-\tilde{V}\left(X_{2}\right) / 2} \sum_{n=0}^{\infty} \psi_{n}\left(X_{2}\right) \psi_{n}\left(X_{0}\right) E_{\alpha}\left(-\lambda_{n, \alpha} t_{2}^{\alpha}\right) \\
& \quad \times \mathrm{e}^{\tilde{V}\left(X_{1}\right) / 2-\tilde{V}\left(X_{0}\right) / 2} \sum_{n=0}^{\infty} \psi_{n}\left(X_{1}\right) \psi_{n}\left(X_{0}\right) E_{\alpha}\left(\lambda_{n, \alpha} t_{1}^{\alpha}\right) \\
&=\mathrm{e}^{\tilde{V}\left(X_{1}\right) /\left(2-\tilde{V}\left(X_{2}\right) / 2\right)} \\
& \quad \times \sum_{n, m=0}^{\infty} E_{\alpha}\left(-\lambda_{n, \alpha} t_{2}^{\alpha}\right) E_{\alpha}\left(\lambda_{n, \alpha} t_{1}^{\alpha}\right) \psi_{m}\left(X_{2}\right) \psi_{n}\left(X_{1}\right) \delta_{n m} \\
&= \mathrm{e}^{\tilde{V}\left(X_{1}\right) / 2-\tilde{V}\left(X_{2}\right) / 2} \sum_{n=0}^{\infty} \psi_{n}\left(X_{2}\right) \psi_{n}\left(X_{1}\right) E_{\alpha}\left(-\lambda_{n, \alpha} t_{2}^{\alpha}\right) E_{\alpha}\left(\lambda_{n, \alpha} t_{1}^{\alpha}\right)
\end{aligned}
$$

where in the last line of eq A7 we used eq 11. Equation A7 is used to compute conditional and joint probabilities in section IV and the three-time correlation function of fluorescence lifetime in section $\mathrm{V}$.

\section{Appendix B: Three-Point Correlation Functions for FRET and ET Quenching}

The $n$-point correlation function of observable $A$ is defined by

$$
\begin{aligned}
\left\langle A\left(t_{n}\right) \ldots\right. & \left.A\left(t_{1}\right) A\left(t_{0}\right)\right\rangle \\
& \equiv \int_{-\infty}^{\infty} \mathrm{d} X_{n} \int_{-\infty}^{\infty} \mathrm{d} X_{n-1} \ldots \int_{-\infty}^{\infty} \mathrm{d} X_{2} \int_{-\infty}^{\infty} \mathrm{d} X_{1} \\
& \times A\left(X_{n}\right) W_{\alpha}\left(X_{n}, t_{n} ; X_{n-1}, t_{n-1}\right) \\
& \times A\left(X_{n-1}\right) W_{\alpha}\left(X_{n-1}, t_{n-1} ; X_{n-2}, t_{n-2}\right) \ldots \\
& \times A\left(X_{1}\right) W_{\alpha}\left(X_{1}, t_{1} ; X_{0}, t_{0}\right) A\left(X_{0}\right) \rho_{\mathrm{eq}}\left(X_{0}\right)
\end{aligned}
$$

Setting $A(X)=\tau(X)$ in the eq B1, we obtain eq 23 for the threepoint correlation function of the fluorescence lifetime. 
For FRET (eq 1), eq B1 gives

$$
\begin{aligned}
& C_{\alpha}^{\mathrm{FRET}}\left(t_{2}, t_{1}\right) \\
& \equiv\left\langle\tau\left(t_{2}\right) \tau\left(t_{1}\right) \tau(0)\right\rangle^{\mathrm{FRET}} \\
& =\left[\frac{1}{2 \pi \theta}\right]^{5 / 4} \sum_{n=0}^{\infty}\left[\frac{1}{2^{n} n !}\right]^{2} E_{\alpha}\left(-n \tilde{t}_{2}^{\alpha}\right) E_{\alpha}\left(n \tilde{t}_{1}^{\alpha}\right) E_{\alpha}\left(-n \tilde{t}_{1}^{\alpha}\right) I_{1}{ }^{2}(n) I_{2}(n)
\end{aligned}
$$

Here $I_{1}(n)$ is given by

$$
I_{1}(n)=\frac{(\sqrt{2 \theta})^{7}}{\gamma_{0} R_{0}^{6}}(-1)^{n / 2} 2^{n} \frac{\Gamma\left(\frac{7}{2}\right) \Gamma\left(\frac{n}{2}+\frac{1}{2}\right)}{\sqrt{\pi}} F\left(-\frac{n}{2}, \frac{7}{2} ; \frac{1}{2} ; 1\right)
$$

where $F(\alpha, \beta ; \gamma ; z)$ is hypergeometric function defined by

$$
F(\alpha, \beta ; \gamma ; z)=\frac{1}{B(\beta, \gamma-\beta)} \int_{0}^{1} \mathrm{~d} t t^{\beta-1}(1-t)^{\gamma-\beta-1}(1-t z)^{-\alpha}
$$

and $B(\beta, \gamma-\beta)$ is beta function, defined by

$$
B(x, y)=\int_{0}^{1} \mathrm{~d} t t^{x-1}(1-t)^{y-1}
$$

$I_{2}(n)$ is given by

$$
\begin{aligned}
I_{2}(n)=\frac{\sqrt{\pi}(\sqrt{2 \theta})^{7}}{\gamma_{0} R_{0}^{6}}\left(10 \frac{2^{n-2}[n !]^{2}}{(n-3) !}-45 \frac{2^{n-2}[n !]^{2}}{(n-2) !}\right. \\
\left.\quad-255 \frac{2^{n-1}[n !]^{2}}{(n-1) !}-255\left(2^{n-2} n !\right)\right)
\end{aligned}
$$

For ET (eq 2), eq B1 gives

$$
\begin{aligned}
& C_{\alpha}^{\mathrm{ET}}\left(t_{2}, t_{1}\right) \\
& =\left\langle\tau\left(t_{2}\right) \tau\left(t_{1}\right) \tau(0)\right\rangle^{\mathrm{ET}} \\
& =\left[\frac{1}{2 \pi \theta}\right]^{5 / 4} \frac{\pi^{3 / 2}}{k_{0}^{2}} \mathrm{e}^{\theta \beta^{2}} \beta^{m+n}(2 \theta)^{m+n+2 / 2} \\
& \times\left(\sum_{m \leq n 2^{m} n !}^{\infty} \frac{1}{\alpha} E_{\alpha}\left(-n \tilde{t}_{2}^{\alpha}\right) E_{\alpha}\left(n \tilde{t}_{1}^{\alpha}\right) E_{\alpha}\left(-m \tilde{t}_{1}^{\alpha}\right)\left(\frac{\beta \sqrt{2 \theta}}{2}\right)^{n-m}\right. \\
& \times L_{m}{ }^{n-m}\left(\beta^{2} \theta\right)+\sum_{m>n}^{\infty} \frac{1}{2^{n} m !} E_{\alpha}\left(-n \tilde{t}_{2}^{\alpha}\right) E_{\alpha}\left(n \tilde{t}_{1}^{\alpha}\right) E_{\alpha}\left(-m \tilde{t}_{1}^{\alpha}\right) \\
& \times\left(\frac{\beta \sqrt{2 \theta}}{2}\right)^{m-n} L_{n}^{m-n}\left(\beta^{2} \theta\right)
\end{aligned}
$$

where $L_{n}{ }^{m}(x)$ is Laguerre polynomial defined by

$$
L_{n}^{m}(x)=\frac{1}{n !} \mathrm{e}^{x} x^{-m} \frac{\mathrm{d}^{n}}{\mathrm{~d} x^{n}}\left[\mathrm{e}^{-x} x^{n+m}\right]
$$

Equations B2 and B7 were used in section V for computing the three-time correlation functions for the FRET and ET quenching mechanisms.

\section{References and Notes}

(1) Nie, S. M.; Zare, R. N. Annu. Rev. Neurosci. 1997, 26, 567.

(2) Xie, X. S.; Trautman, J. K. Annu. Rev. Phys. Chem. 1998, 49, 441.

(3) Tamarat, P.; Maali, A.; Lounis, B.; Orrit, M. J. Phys. Chem. A 2000, 104,1

(4) Moerner, W. E. J. Phys. Chem. B 2002, 106, 910

(5) Fleming, G. R. Chemical Applications of Ultrafast Spectroscopy; Oxford University Press: New York, 1986.

(6) Zwanzig, R. J. Chem. Phys. 1992, 97, 3587; Acc. Chem. Res. 1990 , 23,148 .

(7) Wang, J.; Wolynes, P. Phys. Rev. Lett. 1995, 74, 4317; J. Chem. Phys. 1999, 110, 4812

(8) Chernyak, V.; Schulz, M.; Mukamel, S. J. Chem. Phys. 1999, 111, 7416. Barsegov, V.; Chernyak, V.; Mukamel, S. J. Chem. Phys. 2002, 116, 4240.

(9) Geva, E.; Skinner, J. L. J. Phys. Chem. B 1997, 101, 8920. Geva, E.; Skiner, J. L. Chem. Phys. Lett. 1998, 287, 125.

(10) Berezhkovskii, A. M.; Szabo, A.; Weiss, G. H. J. Chem. Phys. 1999, 110, 9145; J. Phys. Chem. B 2000, 104, 3776.

(11) Yang, S. L.; Cao, J. S. J. Phys. Chem. B 2001, 105, 6536. Cao, J. S. Chem. Phys. Lett. 2000, 327, 38.

(12) Barsegov, V.; Shapir, Y.; Mukamel, S. Phys. Rev. E 2003, 68, 011101.

(13) Fleury, L.; Zambusch, A.; Orrit, M.; Brown, R.; Bernard, J. J. Lumin. 1993, 56, 15. Zambusch, A.; Fleury, L.; Brown, R.; Bernard, J.; Orrit, M. Phys. Rev. Lett. 1993, 70, 3584.

(14) Ambrose, W. P.; Basche, T.; Moerner, W. E. J. Chem. Phys. 1991, 95,7150 .

(15) Barkai, E.; Jung, Y.-J.; Silbey, R. Phys. Rev. Lett. 2001, 87, 207403; Adv. Chem. Phys. 2002, 123, 1 .

(16) Bai, C.; Wang, C.; Xie, X. S.; Wolynes, P. G. Proc. Natl. Acad Sci. U.S.A. 1999, 96, 11075. Xie, X. S. Single Mol. 2001, 2, 229.

(17) Yang, H.; Xie, X. S. J. Chem. Phys. 2002, 117, 10965; Chem. Phys. 2002, 284, 423.

(18) Mandel, L.; Wolf, E. Optical Coherence and Quantum Optics; Cambridge University Press: New York, 1995.

(19) Merz, M.; Schenzle, A. Appl. Phys. 1990, B50, 115.

(20) Zheng, Y.; Brown, F. L. H. Phys. Rev. Lett. 2003, 90, 238305

(21) Edman, L.; Mets, U.; Rigler, R. Proc. Natl. Acad. Sci. U.S.A. 1996, 93, 6710. Eggeling, C.; Fries, J. R.; Brand, L.; Guenter, R.; Seidel, C. A. M. Proc. Natl. Acad. Sci. U.S.A. 1998, 95, 1556. Jia, Y.-W.; Sytnik, A.; Li, L.; Vladimirov, S.; Cooperman, B. S.; Hochstrasser, R. M. Proc. Natl. Acad. Sci. U.S.A. 1997, 94, 7932.

(22) Ha, T.; Ting, A. Y.; Liang, Y.; Caldwell, W. B.; Deniz, A. A.; Chemla, D. S.; Schultz, P. G.; Weiss, S. Proc. Natl. Acad. Sci. U.S.A. 1999, 96, 893. Weiss, S. Science 1999, 283, 1676.

(23) Ha, T.; Zhuang, X.; Kim, H. D.; Orr, J. W.; Williamson, J. R.; Chu, S. Science 1999, 288, 2048. Zhuang, X.; Bartley, L. E.; Babcock, H.

P.; Russell, R.; Ha, T.; Herschlag, D.; Chu, S. Science 2000, 288, 2048. (24) Schlag, E. W.; Sheu, S.-Y.; Yang, D.-Y.; Selzle, H. L.; Lin, S. H. Proc. Natl. Acad. Sci. U.S.A. 2000, 97, 1068.

(25) Lapidus, L. J.; Eaton, W. A.; Hofrichter, J. Proc. Natl. Acad. Sci. U.S.A. 2000, 97, 7220.

(26) Yang, H.; Luo, G.; Karnchanaphanurach, P.; Louie, T.; Xun, L.; Xie, X. S. 2002 (preprint).

(27) Mukamel, S. Principles of Nonlinear Optical Spectroscopy; Oxford University Press: New York, 1995.

(28) Gu, Q.; Schiff, E. A.; Grebner, S.; Schwartz, R. Phys. Rev. Lett. 1996, 76, 3196. Amblard, F.; Maggs, A. C.; Yurke, B.; Pargellis, A. N.; Leibler, S. Phys. Rev. Lett. 1996, 77, 4470.

(29) Bouchaud, J.-P.; Georges, A. Phys. Rep. 1990, 195, 12

(30) Metzler, R.; Barkai, E.; Klafter, J. Phys. Rev. Lett. 1999, 82, 3563.

(31) Metzler, R.; Klafter, J. Phys. Rep. 2000, 339, 1.

(32) Oldham, K. B.; Spanier, J. The Fractional Calculus; Academic Press: New York, 1974

(33) Barsegov, V.; Mukamel, S. J. Chem. Phys. 2002, 116, 9802; 2002, 117,9477

(34) Foerster, T. In Modern Quantum Chemistry, Part III: Action of Light and Organic Molecules; Sinanoglu, O., Ed.; Academic Press: New York, 1965; p 63.

(35) Dexter, D. L. J. Chem. Phys. 1953, 21, 836

(36) Metzler, R.; Klafter, J. J. Phys. Chem. B 2000, 104, 3851.

(37) Risken, H. The Fokker-Planck Equation; Springer-Verlag: Berlin, 1989.

(38) Hansen, E. R. A Table of Series and Products; Prentice-Hall: Englewood Cliffs, NJ, 1975

(39) Sher, H.; Montroll, E. W. Phys. Rev. B 1975, 12, 2455.

(40) Pfister, G.; Sher, H. Phys. Rev. B 1977, 15, 2062.

(41) Zumofen, G.; Blumen, A.; Klafter, J. Phys. Rev. A 1990, 41, 4558.

(42) Weiss, G. H.; Rubin, R. J. Adv. Chem. Phys. 1983, 52, 363.

(43) Shlesinger, M. F. J. Stat. Phys. 1974, 10, 421 